\title{
Impact of a Patient-centered Medical Home on Healthcare Utilization for Patients With Complex Needs in Singapore: a Matched Cohort Study
}

Silvia Yu Hui Sim ( $\square$ sim.silvia@gmail.com )

Geriatric Education \& Research Institute https://orcid.org/0000-0001-5196-8271 Junxing Chay

Duke-NUS Medical School https://orcid.org/0000-0002-3291-4523

Soon Hoe Ho

Geriatric Education \& Research Institute

Mimaika Luluina Ginting

Geriatric Education \& Research Institute https://orcid.org/0000-0002-0319-8097

Zoe Zon Be Lim

Geriatric Education \& Research Institute https://orcid.org/0000-0002-8256-9924

Grace Sum

Geriatric Education \& Research Institute https://orcid.org/0000-0002-0309-2700

Joanne Yoong

University of Southern California https://orcid.org/0000-0002-0162-9885

Chek Hooi Wong

Tsao Foundation https://orcid.org/0000-0002-3048-0477

\section{Research Article}

Keywords: Patient-Centered Medical Home, complex needs, primary care, healthcare utilization

Posted Date: February 18th, 2022

DOI: https://doi.org/10.21203/rs.3.rs-1151743/v1

License: (1) (1) This work is licensed under a Creative Commons Attribution 4.0 International License. Read Full License 


\section{Abstract}

Background: Patients with complex needs require greater biopsychosocial support. Fragmented services often result in gaps, duplication or incompatibility in treatments, leading to poorer health outcomes and higher utilization of costly hospital-based healthcare services. Patient-Centred Medical Home (PCMH) is a primary care model reform advocated as more appropriate for patients with complex needs. This analysis assessed the impact of a PCMH demonstration on healthcare utilization for patients with complex needs in Singapore.

Methods: This study assessed the Community for Successful Ageing (ComSA)-PCMH in Singapore that was launched in Nov'2016. ComSA-PCMH serves adults aged 40 and above with complex needs, delivering care integrated between its primary care clinic, home-based care management, an acute hospital and other community-based services.

This was a matched cohort analysis that used a difference-in-difference approach to compare quarterly healthcare utilization of PCMH study participants and controls. $165 \mathrm{PCMH}$ study participants enrolled between Oct'2017 and Apr'2019 were compared against 5,385 controls selected using Coarsened Exact Matching from a neighbouring geographical precinct. Healthcare utilization data spanning Oct'2014 to Mar'2020 were extracted from a public regional health system, including primary care, specialist outpatient clinic and emergency department utilization, and inpatient admissions.

Two-part models were fitted, with controls for socio-demographics, Charlson Comorbidity Index, secular trends including effect of COVID-19, and between-group differences. Statistical inferences were based on cluster-robust standard errors at the individual level.

Results: At $\geq 4$ four quarters post-enrolment, PCMH study participants had sustained reductions of 1.08 polyclinic visits $(p<0.001), 0.37$ SOC visits $(p=0.047), 0.04$ ED visits $(p=0.013)$ and 0.02 inpatient admissions $(p=0.093)$ per person-quarter, compared to the quarter before enrolment and controls.

Conclusions: ComSA-PCMH study participants reduced healthcare utilization after enrolment into the programme, adding to a growing global body of evidence supporting the benefits of PCMH for patients with complex needs and pointing to its potential in Asia.

Trial registration: This analysis is part of the ComSA Patient-Centered Care (COPACC) study, retrospectively registered with ClinicalTrials.gov in October 2020 (Protocol ID: NCT04594967; https://clinicaltrials.gov/ct2/show/NCT04594967).

\section{Background}

Globally, there is a rapid increase in the prevalence of multiple chronic conditions, which is associated with poorer physical function and quality of life, increased risk of morbidity, disability, and psychological stress.' ' It is increasingly acknowledged that a fragmented health system comprised of disease-specific 
models of care is inadequate in managing these needs. 'When multiple conditions are managed in silos, issues such as unnecessary polypharmacy, adverse drug interactions and treatment gaps tend to arise. ${ }^{5}$ Fragmented care may also overlook non-medical influences that add complexity to the management of these patients, including socio-economic challenges, family stressors and mental health issues such as depression. This adds burden to patients and caregivers by requiring them to navigate between multiple service providers and reconcile various treatment and services, resulting in patients with complex needs falling through the cracks and/or presenting to inappropriate healthcare settings. Studies have shown that patients with multiple chronic conditions are far more likely to incur hospital admissions for ambulatory care-sensitive conditions, and found a curvilinear, almost exponential relationship between multiple chronic conditions and healthcare utilization and costs.

Singapore, a small developed country in Asia, faces similar challenges to its health system with increasing numbers of patients with complex needs. It is estimated that 1 out of 5 Singaporeans will be aged 65 and above by year 2030. The ageing trend is accompanied by increasing chronic disease burden. The proportion of Singaporeans with $\geq 3$ chronic conditions rose from 19.8-37.6\% within a decade, from 2009 to 2017. Over the same period, those with functional difficulties in $\geq 1$ basic activity of daily living (ADL) rose from $6.3-9.3 \%$, and those with difficulties in $\geq 3$ instrumental activities of daily living (IADL) rose from $6.4-8.3 \%$. Like most developed countries, Singapore's healthcare system was originally designed for the acute, episodic healthcare needs of a younger population of the past. ${ }^{6}$ With the evolving demographics and healthcare needs, it is acknowledged that a stronger primary care model is required to provide more appropriate care.

The Patient-Centered Medical Home (PCMH), a primary care model, has been advocated as a more appropriate model for patients with complex needs. ${ }^{8}$ Contrary to disease-specific models of care, the PCMH model aims for comprehensive management of healthcare needs, recognizing the interactions between various medical conditions and non-medical challenges such as social and financial needs. This is accomplished through improved communication and coordination between the patient, family and service providers. ${ }^{8}$ The PCMH model has been shown to reduce inappropriate hospital-based healthcare utilization with improved management of care needs in the primary care setting and coordination of referrals to more appropriate care settings.'

Most evaluation studies have found the PCMH model to be most effective in populations that are older or have higher needs, resulting in better health outcomes and reduced hospital-based healthcare utilization.' '..' In contrast, studies that found non-significant changes tend to be amongst populations with lower needs, or examine very early stages of PCMH implementation. ${ }^{21}$, Moreover, implementation and evaluation of PCMH models have been carried out predominantly in the West, while less is known about its effectiveness in an Asian context.

This study aims to assess the impact of a PCMH demonstration on healthcare utilization for patients with complex needs in Singapore. This, to the authors' knowledge, is the first study to evaluate the impact 
of PCMH on healthcare utilization in Singapore and in Asia. This study would address the existing knowledge gaps about the effectiveness of PCMH in an Asian context and contribute to the wider discussion on the delivery of primary care for adults with complex needs.

\section{Methods}

\subsection{Study design}

This is a matched cohort study using a difference-in-difference approach to analyse healthcare utilization of PCMH study participants.

\subsection{Intervention}

This study assessed the Community for Successful Ageing (ComSA)-PCMH, launched in November 2016 in a geographically defined central region of Singapore. Adults aged 40 and above identified to have high biopsychosocial needs by a locally-validated risk screening tool or clinical judgment, and residing within the geographic area were recruited to the clinic. These included referrals from specialist clinics and primary care clinics from the public regional health system, other private primary care and community partners as well as patients who walked in without referrals.

ComSA-PCMH consists of a primary care clinic led by family doctors trained in geriatric primary care and care coordination, as well as a home-based care management service led by medical social workers and nurses to support adherence to care plan, care coordination to manage financial problems, health behaviour challenges, family conflict, caregiver stress and social engagement. The initial visits comprise a comprehensive needs assessment and care plan development with patient and caregivers. This is followed by regular reviews and acute treatments if needed. ComSA-PCMH delivers care that is integrated between the primary care clinic, home-based care management, the referring acute hospital and other community-based services. Patients are empanelled within the integrated PCMH with a regular primary care physician and home care team for lifelong engagement, to facilitate continuity of care and sustained patient-provider relationship. Patients are only discharged if they had no contact with ComSA-PCMH for $>18$ months, became institutionalised in nursing homes, homebound or passed away.

\subsection{Sample}

This study recruited 184 patients within their initial visits to ComSA-PCMH, from October 2017 to April 2019. Controls were selected from a neighbouring geographical region with similar population-level demographics of age, ethnicity, housing type and disease prevalence, and is served by the same public regional health system that did not offer the PCMH model of care. As participants had complex medical

and non-medical needs, the sampling frame for controls was derived from the administrative records of a wide range of healthcare services, namely five public primary care clinics (termed 'polyclinics' in Singapore) and specialist outpatient clinics (SOC), emergency department (ED) and impatient services under the public regional health system, from 1 September 2014 to 31 March 2020. The sampling frame 
consisted of 11,269 unique persons residing in the control region. Persons who had passed away during the period of analysis or had incomplete data were excluded for a complete case analysis (Figure 1).

Matched controls were selected using Coarsened Exact Matching (CEM) on variables that may affect healthcare utilization patterns: birth year, gender, housing type, ethnicity, weighted Charlson Comorbidity Index (CCl) and whether the person had any inpatient admission or specialist outpatient clinic visit in the quarter before enrolment. Variables were temporarily coarsened into substantively meaningful groups (Table 1) and all exact matches were identified from the control group. Original, un-coarsened values were retained for analysis. CEM was selected over Propensity Score Matching as it can achieve lower levels of covariate imbalance, model dependence and bias. This matching method yielded different proportions of PCMH study participants and controls across the different coarsened typologies. All matched units were included in the analysis and weighting was used to account for the unequal strata sizes. The final sample consisted of $n=165$ PCMH study participants and $n=5,385$ controls (Figure 1 ).

Table 1

Variables involved in Coarsened Exact Matching

\begin{tabular}{|c|c|}
\hline Variable & Coarsened categories \\
\hline Birth year & $\leq 1940,1941-1950,1951-1960,1961-1970, \geq 1971$ \\
\hline Gender & Male, Female \\
\hline \multirow[t]{2}{*}{ Ethnicity } & Chinese, Malay, Indian \& Sikh \\
\hline & Based on Singapore's ethnic categorization \\
\hline Housing type & Public, Private \\
\hline \multirow[t]{3}{*}{$\mathrm{CCl}$} & $0,1-2,2-4, \geq 5$ \\
\hline & Based on categorization by Menendez et al. 2014 \\
\hline & $\begin{array}{l}\text { Note: Calculation for PCMH study participants based on individual } \\
\text { enrolment date. Calculation for controls based on earliest } \\
\text { enrolment date into ComSA-PCMH amongst study participants }\end{array}$ \\
\hline $\begin{array}{l}\text { Had inpatient admission or } \\
\text { specialist outpatient clinic } \\
\text { visits in the quarter before } \\
\text { enrolment }\end{array}$ & Yes, No \\
\hline
\end{tabular}

\subsection{Outcome measures}

The study assessed outcomes in healthcare utilization at public healthcare providers. Public providers are major players in Singapore's healthcare scene, with polyclinics serving approximately $40 \%$ of chronic disease-related visits in primary care and public hospitals providing approximately $80 \%$ or more of tertiary inpatient services.' 
Healthcare utilization outcomes assessed were polyclinic visits, SOC visits, ED visits and inpatient admissions. We included visits from 1 October 2014 to 31 March 2020, to cover at least 3 years before the first PCMH study participant's enrolment and 1 year after the last study participant's enrolment into $\mathrm{PCMH}$.

\subsection{Data sources}

The study extracted administrative records from a public regional health system. This included records of polyclinic visits, SOC visits, ED visits, inpatient admissions and demographic data. From these records, we calculated the unique number of visits per person, per calendar-quarter (i.e. person-quarter) and $\mathrm{CCl}$ for all PCMH study participants and controls.

For study participants who were not in the public regional health system's administrative records, we derived demographic data and $\mathrm{CCl}$ from the PCMH clinic administrative database.

\subsection{Data analysis}

Difference-in-differences was used to identify changes in healthcare utilization of PCMH study participants pre- and post-enrolment into $\mathrm{PCMH}$, relative to matched controls. This design enables assessment of whether enrolment into PCMH was associated with changes in healthcare utilization over time that were statistically different from the secular trend.

Regression analysis was conducted by fitting a two-part model for each outcome measure. For the first part, a logistic regression model was fitted where the dependent variable was whether there was any (i.e. $\geq 1$ ) healthcare utilization event (binary yes/no) per person-quarter. For the second part, a Poisson regression was fitted, where the dependent variable was the number of healthcare utilization events per study person-quarter, among those who had any healthcare utilization events. This was conducted to handle the high number of person-quarters with zero healthcare utilization events in the data."

The main independent variable assessed is time since enrolment into PCMH. This was modelled using dummy variables to identify any non-linear effects over time. We included individual controls for each quarter, up to 7 quarters pre-enrolment and 3 quarters post-enrolment. Effects for time periods 8 quarters or more pre-enrolment and 4 quarters or more post-enrolment were treated as constant and grouped. In this analysis, enrolment refers to the calendar quarter (3 months) in which the PCMH study participant was enrolled into $\mathrm{PCMH}$. Baseline referred to the quarter before enrolment. Controls were never enrolled into PCMH and hence took zero values for all dummy variables indicating time since enrolment.

Potential confounders were controlled for in both parts of the model: birth year, gender, ethnicity, housing type, $\mathrm{CCl}$ and treatment group ( $\mathrm{PCMH}$, control) to account for any unobserved difference between groups. Calendar time was also adjusted for, to account for secular changes in healthcare utilization, modelled using dummy variables to identify any non-linear trends. The last calendar quarter in this analysis (i.e. 2020Q1) is of particular interest as COVID-19 had spread to Singapore during that period. An interaction term $(2020 \times \mathrm{PCMH})$ was included to account for any differential effects that PCMH study participants 
sustained in 2020Q1 compared to controls. Cluster-robust standard errors at person-level were used to account for potential within-person correlation of healthcare utilization over time.

The adjusted average marginal effects which combines Part 1 (logistic) and Part 2 (Poisson) were calculated.

\section{Results}

\subsection{Descriptive statistics}

PCMH study participants had a mean age of 76 years [SD=9.61] and mean age-adjusted $\mathrm{CCl}$ of 4.51 [SD $=2.01$ ] as of 2017 , which were not statistically significantly different from controls (Table 2). Both PCMH study participants and controls had similar prevalence of diabetes with and without complications, cerebrovascular disease and renal disease as comorbidities, although PCMH study participants had significantly higher prevalence of dementia $\left(\chi^{2}=3.39, p<0.001\right)$ compared to controls (Figure 2). 
Table 2

Post-matching case-control characteristics

\begin{tabular}{|c|c|c|c|}
\hline Variable & $\begin{array}{l}\text { PCMH Study } \\
\text { Participants }(n=165)\end{array}$ & $\begin{array}{l}\text { Controls }(n=5385, \\
\left.\text { weighted }^{1}\right)\end{array}$ & $\begin{array}{l}\text { t-test score } \\
\text { (2-tailed, } \\
\text { weighted }^{1} \text { ) }\end{array}$ \\
\hline $\begin{array}{l}\text { Age (mean [SD], as of } \\
\text { 2017) }\end{array}$ & $76[9.61]$ & 75 [9.03] & $\begin{array}{l}t(173)=-0.60 \\
p=0.55\end{array}$ \\
\hline \multirow[t]{3}{*}{ Gender, Female (\%) } & 55.8 & 55.8 & \multirow{7}{*}{$\begin{array}{l}\text { NA due to exact } \\
\text { matching }\end{array}$} \\
\hline & 92.7 & 99.4 & \\
\hline & 2.4 & & \\
\hline Ethnicity, Chinese (\%) & 92.7 & 92.7 & \\
\hline Ethnicity, Malay (\%) & 2.4 & 2.4 & \\
\hline Ethnicity, Indian \& Sikh (\%) & 4.8 & 4.8 & \\
\hline Housing, Public (\%) & 99.4 & 99.4 & \\
\hline $\begin{array}{l}\text { Age-adjusted CCl } \\
\text { (mean [SD]) }\end{array}$ & $4.51[2.01]$ & $4.53[2.83]$ & $\begin{array}{l}t(176)=-0.14 \\
p=0.89\end{array}$ \\
\hline $\begin{array}{l}\text { Baseline no. of polyclinic } \\
\text { visits } \\
\text { (mean [SD], per person- } \\
\text { quarter) }\end{array}$ & $0.61[1.02]$ & $0.88[1.16)$ & $\begin{array}{l}t(177)=-3.25 \\
p=0.001\end{array}$ \\
\hline $\begin{array}{l}\text { Baseline no. of SOC visits } \\
\text { (mean [SD], per person- } \\
\text { quarter) }\end{array}$ & $1.47[2.17]$ & $1.22[1.78]$ & $\begin{array}{l}t(171)=1.48 \\
p=0.14\end{array}$ \\
\hline $\begin{array}{l}\text { Baseline no. of ED visits } \\
\text { (mean [SD], per person- } \\
\text { quarter) }\end{array}$ & $0.16[0.55]$ & $0.08[0.17]$ & $\begin{array}{l}t(165)=1.88 \\
p=0.06\end{array}$ \\
\hline $\begin{array}{l}\text { Baseline no. of inpatient } \\
\text { admissions } \\
\text { (mean [SD], per person- } \\
\text { quarter) }\end{array}$ & $0.11[0.37]$ & $0.05[0.13]$ & $\begin{array}{l}t(165)=1.95 \\
p=0.05\end{array}$ \\
\hline
\end{tabular}

At baseline, both PCMH study participants $(\mathrm{P})$ and controls(C) had highest utilization in SOC (P:1.47visits/person-quarter; C:1.22visits/person-quarter), followed by polyclinics (P:0.61visits/person- 
quarter; C:0.88visits/person-quarter), ED (P:0.16visits/person-quarter; C:0.08visits/person-quarter) and inpatient admissions (P:0.11visits/person-quarter; $\mathrm{C}: 0.05 \mathrm{visits} /$ person-quarter). $\mathrm{PCMH}$ study participants had significantly fewer polyclinic visits $(\mathrm{t}(177)=-3.25, p=0.001)$ and marginally more ED visits $(\mathrm{t}(165)=1.88, p=0.06)$ and inpatient admissions $(\mathrm{t}(165)=1.95, p=0.05)$ per person-quarter compared to controls. (Table 2).

\subsection{Difference-in-difference}

Figures 3-6 plot the marginal effects of the two-part model for polyclinic visits, SOC visits, ED visits and inpatient admissions respectively.

Relative increases in the healthcare utilization of PCMH study participants compared to controls were observed over the pre-enrolment quarters. Then, relative reductions were first observed in polyclinic visits in the quarter before enrolment, followed by SOC visits two quarter after enrolment and ED visits three quarters after enrolment, continuing till the end of the analysis period.

At $\geq 4$ quarters post-enrolment, $\mathrm{PCMH}$ study participants had sustained significant reductions of 1.08 polyclinic visits (dy/dx: $-1.08, p<0.001), 0.37$ SOC visits (dy/dx: $-0.37, p=0.047), 0.04 \mathrm{ED}$ visits (dy/dx: $-0.04, p=0.013)$, and showed marginally-significant reductions of 0.02 inpatient admissions per personquarter (dy/dx: $-0.02, p=0.093)$, compared to the quarter before enrolment and controls. Estimates of the full model are reported in the Appendices.

\section{Discussion}

Reductions in the healthcare utilization assessed were observed after enrolment into PCMH. These effects were robust even after adjusting for potential confounders, secular trends and differential changes in healthcare utilization that PCMH study participants and controls might have during the first quarter of Covid-19 outbreak in Singapore.

At $\geq 4$ four quarters post-enrolment, $\mathrm{PCMH}$ study participants had sustained reductions of 1.08 polyclinic visits $(p<0.001), 0.37$ SOC visits $(p=0.047), 0.04$ ED visits $(p=0.013)$ and 0.02 inpatient admissions $(p=0.093)$ per person-quarter, compared to the quarter before enrolment and controls.

These findings are aligned with previous evaluation studies that also found reductions in SOC visits, ED visits and inpatient admissions associated with PCMH interventions for patients with higher needs. ${ }^{15,16}$, $17,18,19,20,21,22$. Possible mechanisms for reduction in hospital-based healthcare utilization could be explained by the qualitative findings of the larger study. Participants and caregivers reported that they were able to consolidate care at $\mathrm{PCMH}$, as they received comprehensive care at $\mathrm{PCMH}$ that was of similar or better quality compared to their previous usual providers. The positive care experience was attributed to the continuity, personalisation and holistic management of care, and a sustained patient-provider relationship. These findings were triangulated by findings reported in other studies. A qualitative study with patients, payers, implementation staff and experts identified that continuity of care and adoption of 
care plan were amongst high-value elements that reduce healthcare utilization. Another cohort study observed that patients with continuity of care were less likely to have ED visits.

The one-year time lag before significant reductions was also consistent with the literature. A previous randomized controlled trial in the United States found that amongst patients at high risk for hospitalization, non-significant changes were observed in the first year but significant reductions in inpatient admissions and ED visits were observed in the second year. The authors proposed that a period of engagement was needed to develop trust between the care team and the patients, before patients would reduce utilization of other healthcare services. ${ }^{16}$

This study had various strengths. Firstly, this study used administrative data from a reliable public regional health system to calculate healthcare utilization. This afforded good coverage of the study participants' healthcare utilization, as public providers are major players in Singapore's healthcare scene, with public hospitals providing approximately $80 \%$ or more of tertiary inpatient services. ${ }^{29}$ Administrative data also provided an accurate and objective measure that is not affected by recall error or other response biases.

Secondly, this analysis used a difference-in-difference approach. This design enabled assessment of whether enrolment into PCMH was associated with changes in healthcare utilization over time that were statistically different from the secular trend, combining the strengths of a case-control comparison and cohort study.

Thirdly, analysis of quarterly healthcare utilization provided more granular insights compared to commonly-seen measures of yearly healthcare utilization, while retaining sufficient aggregation to tolerate 'noise' in the data.

There were also some limitations to this study. Firstly, the controls are matched only on available observables, and hence may not be ideal counterfactuals, as we did not have sufficient data to match on psychological and social needs. Several indicators suggest that PCMH study participants had higher needs than controls. For example, PCMH study participants had significantly fewer polyclinic visits and more inpatient admissions and ED visits at baseline, compared to the controls. The higher inpatient admissions and ED visits at baseline were contributed by increases over 8 quarters or more before enrolment. This points to a prolonged increase in complexity of medical needs rather than an acute crisis episode, and is unlikely to be followed by recovery with usual care. . In addition, PCMH study participants had a higher prevalence of dementia (P:18\%; C:6\%), which is strongly associated with higher long-term psychosocial needs and healthcare utilization.' Accordingly, relative improvements after enrolment into PCMH would likely be larger if compared to a group with more similar needs.

Causal inference of the estimated reduction of polyclinic visits should also be interpreted with caution, since a small, marginally-significant reduction was observed even before enrolment into $P C M H\left(Q_{-2}\right.$ $\mathrm{dy} / \mathrm{dx}: 0.24, p=0.076)$. This reduction occurred with concomitant increases in SOC visits, inpatient admissions and ED visits before enrolment. As ComSA-PCMH enrolled patients with complex care needs, 
it could be postulated that by the time of enrolment, polyclinic visits might have become inadequate to support the increase in complexity of care needs. Some care substitution with hospital services may have occurred.

In addition, this analysis did not differentiate between avoidable and unavoidable hospital visits. Avoidable visits include those due to social reasons or milder conditions that could be treated in primary care, or due to escalations that could have been avoided if the conditions had been better managed in primary care. As such, the reductions for avoidable visits could be expected to be more pronounced compared to reductions in all hospital visits, as PCMH is expected to reduce hospital visits via improved management of ambulatory-care sensitive conditions as well as consolidation and coordination of care. ${ }^{15}$ This can be assessed in future research to improve understanding of the mechanisms that contributed to the reductions in health utilization.

Lastly, this analysis assessed healthcare utilization in a limited time period post-enrolment. Our findings suggest that a longer follow-up period may allow us to capture more comprehensive effects.

\section{Conclusions}

Our study findings support the hypothesis that enrolment into PCMH is associated with reductions in hospital-based healthcare utilization. While further research is needed to assess its longer-term effectiveness, sustainability and generalizability to other parts of Asia, these findings add to a growing body of evidence supporting the benefits of PCMHs for adults with complex needs globally and point to the potential of PCMH in Asia.

\section{Abbreviations}

ADL: Activities of Daily Living

C: Controls

CCI: Charlson Comorbidity Index

CEM: Coarsened Exact Matching

ComSA: Community for Successful Ageing

ED: Emergency Department

IADL: Activities of Daily Living

P: Participants

PCMH: Patient-Centered Medical Home 


\section{Declarations}

\section{i. Ethics Approval}

This study was approved by the Domain Specific Review Board (DSRB) of National Healthcare Group (NHG), Singapore (protocol ID 2017/00352, approved 1 August 2017). The Domain Specific Review Board's policies are based on local and international ethical guidelines, including Belmont Report, Declaration of Helsinki, and Ministry of Health Singapore Code of Ethical Practice in Human Biomedical Research.

NHG DSRB approved a waiver of consent for extraction of administrative data from the public regional health system, as study findings would not change the care which the participants would have already received. Written informed consent was obtained from study participants who were not in the public regional health system's administrative records, for extraction of their records from the PCMH clinic administrative database.

\section{ii. Consent for publication}

Not applicable - this manuscript does not contain any personally-identifiable information.

\section{iii. Availability of data and materials}

The data presented in this study are not publicly available to protect patients' privacy.

\section{iv. Competing interests}

$\mathrm{CHW}$ is currently employed at Tsao Foundation. The employment commenced after the completion of the study and analysis, and did not have any impact on manuscript preparation. All other authors had no conflict of interests relating to the subject matter discussed in this manuscript.

\section{v. Funding}

This study was funded by the Geriatric Education and Research Institute Intramural Research Grant (GERI1608). The funder played no part in study design, data collection, analysis, interpretation, or manuscript writing.

\section{vi. Authors' contribution}


CHW was principal investigator, while MLG and ZZBL were co-investigators of the study. SYHS and SHH analysed and interpreted the data with advice from JC, JY and all authors. SYHS wrote the first draft of the manuscript and subsequently edited with inputs from all authors JC, SHH, MLG, ZZBL, GS, JY and CW. All authors read and approved the final manuscript.

\section{vii. Acknowledgments}

We would like to thank:

- Our implementation partner Tsao Foundation, in particular Dr. Mary Ann Tsao, Dr. Sai Tiang Tan, Dr. Wai Chong $\mathrm{Ng}$ and Moana Jagasia;

- National Healthcare Group for providing administrative data, in particular Eugene Yew Chung Yuen;

- Other researchers on the study team, including Bing Xie, Mumtaz Mohamed Kadir, Robin Wai Munn Choo and Sheena Camilla Hirose Carlsen;

- Our study administrators Isabelle Shu Fen Lim and Julian Zhi Liang Loke;

- Last but not least, our study participants.

\section{References}

1. Hajat C. and Stein E. The global burden of multiple chronic conditions: A narrative review. Preventive Medicine Reports 2018; 12:284-293

2. Vos T, Lim SS, Abbafati C et al. Global burden of 369 diseases and injuries in 204 countries and territories, 1990-2019: A systematic analysis for the Global Burden of Disease Study 2019. The Lancet 2020; 396:10258.

3. Fortin M, Lapointe L, Hudon $\mathrm{C}$ et al. Multimorbidity and quality of life in primary care: A systematic review. Health and Quality of Life Outcomes 2004; 2(51).

4. Megari K. Quality of life in chronic disease patients. Health Psychology Research 2013; 1: e27.

5. Moffat K, Mercer SW. Challenges of managing people with multimorbidity in today's healthcare systems. BMC Fam Pract 2015; 16:129

6. Ong B. The future of Singapore healthcare. Medicine (Yong Loo Lin School of Medicine) Issue 20. 2016. https://medicine.nus.edu.sg/newsletter/issue-20/insights/the-future-of-singapore-healthcare/ (Accessed Jun 8, 2021)

7. Loeb DF, Binswanger IA, Candrian C and Bayliss EA. Primary care physician insights into a typology of the complex patient in primary care. Annals of Family Medicine 2015; 13(5).

8. Mathematica Policy Research. Coordinating care for adults with complex care needs in the patientcentered medical home: challenges and solutions. Rockville, MD: Agency for Healthcare Research and Quality, 2012. 
9. Wolff JL, Starfield B and Anderson G. Prevalence, expenditures and complications of multiple chronic conditions in the elderly. JAMA Internal Medicine 2002; 162(20).

10. Lehnert T, Heider D, Leicht H, Heinrich S, Corrieri S, Luppa, Riedel-Heller S, Konig H-H. Review: Health Care Utilization and Costs of Elderly Persons with Multiple Chronic Conditions. Medical Care Research and Review 2011; 68(4).

11. Wong $\mathrm{CH}$ and Landefeld CS. Academic Geriatrics in Singapore. Journal of the American Geriatrics Society 2011; 59(11).

12. Malhotra, R. and Chan, A. PHASE and THE SIGNS Study: An overview of two nationally representative surveys of older adults in Singapore. Regional workshop on Integrating Policy and Research Ageing in ASEAN: Conversations across the Policy and Research, 4 to 6 Sep 2019. 2019. https://duke-nus.edu.sg/docs/librariesprovider3/education-docs/1)-phase-and-the-signsstudy-by-assistant-prof-rahul-malhotra-(singapore)935707bf622d4cd28c4a00b3b7872b57.pdf? sfvrsn=d5d2f439_0 (accessed 8 Jun 2021).

13. Ministry of Health. Opening address by Dr Amy Khor, Senior Minister of State, Ministry of Health, at the Singapore Healthcare Management Congress 2018, 14 August 2018. https://www.moh.gov.sg/news-highlights/details/opening-address-by-dr-amy-khor-seniorminister-of-state-ministry-of-health-at-the-singapore-healthcare-management-congress-2018-14august-2018/ (accessed 8 Jun 2021)

14. Grant RW, Jeffrey MA, Hong CC et al. Defining patient complexity from the primary care physician's perspective. Annals of Internal Medicine 2011; 155: 797-804.

15. David G, Gunnarsson C, Sayniscsh PA et al. Do Patient-Centered Medical Homes reduce emergency department visits? Health Services Research 2015; 50(2).

16. Counsell S, Callahan C, Clark D et al. Geriatric care management for low-income seniors - A randomised-controlled trial. JAMA 2007; 298(22).

17. Flottemesch TJ, Anderson LH, Solberg LI et al. Patient-centered medical home cost reductions limited to complex patients. The American Journal of Managed Care 2012; 18(11): 677-686.

18. Jackson GL, Powers BJ, Chatterjee R et al. The Patient-Centered Medical Home: A systematic review. Annals of Internal Medicine 2013; 158:169-178.

19. Rosenthal 2013 Effect of a multipayer PCMH on healthcare utilization and quality: The Rhode Island chronic care sustainability initiative pilot. JAMA Intern Med 2013; 173(20): 1907-1913.

20. Neal J, Chawla R, Colombo CM et al. Medical Homes: Cost effects of utilization by chronically-ill patients. The American Journal of Managed Care 2015; 21(1): e51-e61.

21. The National Committee of Quality Assurance. Benefits of NCQA Patient-Centered Medical Home Recognition. Washington, DC: The National Committee of Quality Assurance, 2019. https://ncqa.org/wp-content/uploads/2019/09/20190926_PCMH_Evidence_report.pdf (Accessed Oct 1, 2020).

22. John J, Jones A, Neville A, et al. Cohort profile: effectiveness of a 12-month patient-centered medical home model versus standard care for chronic disease management among primary care patients in 
Sydney, Australia. International Journal of Environmental Research and Public Health 2020; 17: 2164.

23. Friedberg MW, Schneider EC, Rosenthal MB et al. Association between participation in a multipayer medical home intervention and changes in quality, utilization and costs of care. JAMA 2014; 311(8):815-825.

24. Surendran S, Foo C, Tam CH, et al. The Missed Opportunity of Patient-Centered Medical Homes to Thrive in an Asian Context. Int J Environ Res Public Health 2021; 18(4).

25. Hildon ZJ-L, Tan CS, Shiraz F, Ng WC, Deng X, Koh GCH, et al. The theoretical and empirical basis of a BioPsychoSocial (BPS) risk screener for detection of older people's health related needs, planning of community programs, and targeted care interventions. BMC Geriatrics 2018;18(49).

26. Bodenheimer $T$ et al. The 10 Building Blocks of High-Performing Primary Care. The Annals of Family Medicine 2014; 12(2): 166-171.

27. King G, Nielsen R. Why propensity scores should not be used for matching. Political Analysis 2019; 27: 435-454.

28. Ministry of Health, Health Information Division. MOH Primary Care Survey. 2014. https://www.moh.gov.sg/docs/librariesprovider5/resources-statistics/reports/moh-primary-caresurvey-2014-report.pdf (Accessed November 20, 2020)

29. Khoo L. MOH information paper: Singapore healthcare market-share analysis. 2003. https://www.moh.gov.sg/docs/librariesprovider5/resources-statistics/informationpapers/singapore_healthcare_market-share_analysis.pdf (Accessed November 20, 2020)

30. Kaushal R, Edwards A, Kern LM. Association between the patient-centered medical home and healthcare utilization. American Journal of Managed Care 2015; 21(5).

31. Belotti F, Manning WG, Deb P et al. Two-part models. The Stata Journal 2016; 15: 3-20.

32. Ahn S, Hussein M, Mahmood A et al. Emergency department and inpatient utilization among U.S. older adults with multiple chronic conditions: a post-reform update. BMC Health Services Research 2020; 20(77).

33. Rosenthal $M$, Alidina $S$, Friedberg $M$ et al. A difference-in-difference analysis of changes in quality, utilization and cost following the Colorado multi-payer Patient-Centered Medical Home pilot. Journal of General Internal Medicine 2015; 31: 289-296.

34. Ginting ML, Lim ZZB, Carlsen SCH et al. A first demonstration of the Patient-Centered Medical Home $(\mathrm{PCMH})$ care model in Singapore: A qualitative study on patient's experience. $21^{\text {st }}$ International Conference on Integrated Care 2021 (ICIC21) Virtual Conference; 2021 May 27. Available from: https://abbey.eventsair.com/QuickEventWebsitePortal/icic21virtual/programme/Agenda/AgendaltemDetail?id=a5a4c881-e7ab-4215-bcf6-5f7183289d27

35. Anastas TM, Wagner J, Ross RL et al. Defining high value elements for reducing cost and utilization in Patient-Centered Medical Homes for the TOPMED trial. EGEMS 2019; 7(1): 20. 
36. Chaiyachati $\mathrm{KH}$, Gordon $\mathrm{K}$, Long $\mathrm{T}$ et al. Continuity in a VA patient-centered medical home reduces emergency department visits. PLOS One 2014; 9(8).

37. Morton V. Effect of regression to the mean on decision-making in healthcare. BMJ 2003; 326: 10831084.

38. Whitlatch CJ and Orsulic-Jeras S. Meeting the informational, educational and psychosocial support needs of persons living with dementia. The Gerontologist 2018; 58(S1): S58-S73.

39. Chung SD, Liu SP, Sheu JJ et al. Increased healthcare service utilizations for patients with dementia: A population-based study. Plos One 2014; 9(8).

\section{Figures}


184 PCMH study participants

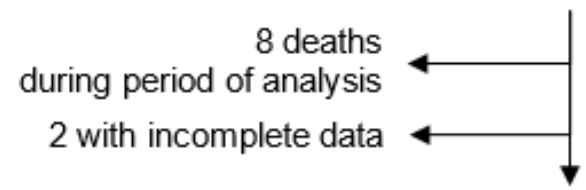

$165 \mathrm{PCMH}$ study participants
11,269 residents in control region

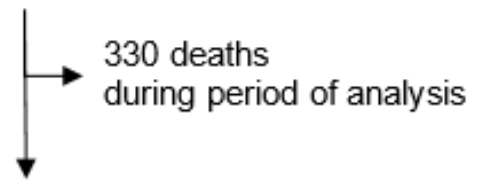

10,939 residents in control region

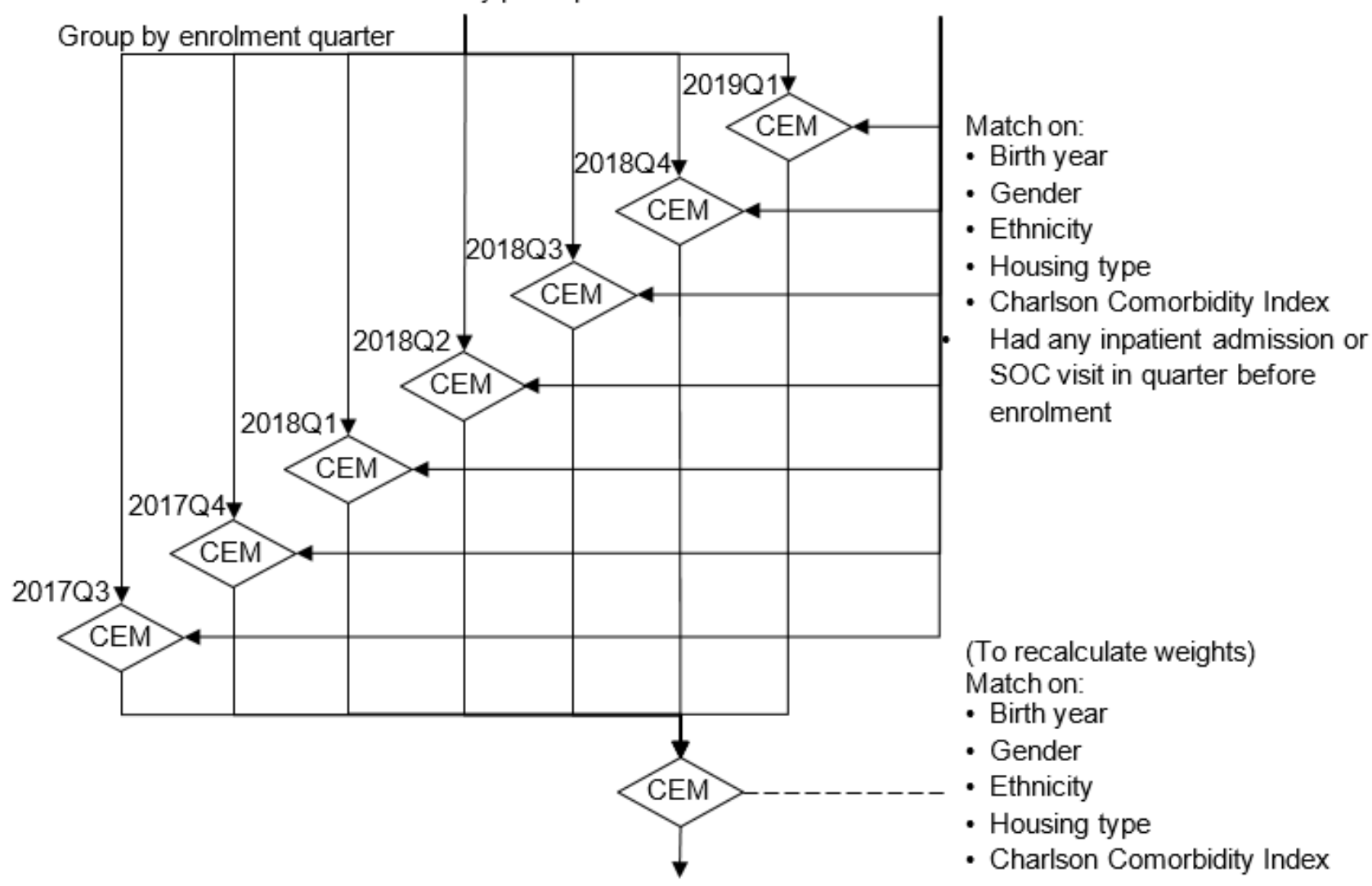

165 PCMH study participants 5,385 matched controls

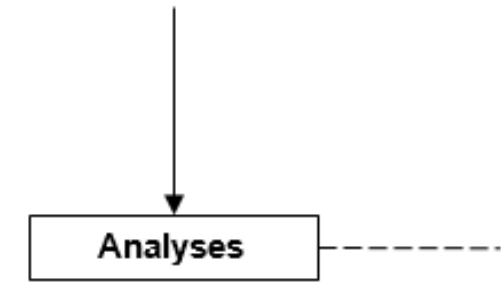

Healthcare utilization assessed:
a. Polyclinic visits
b.SOC visits

c. Inpatient Admissions

d. ED visits

\section{Figure 1}

Study Flow for study sample selection 


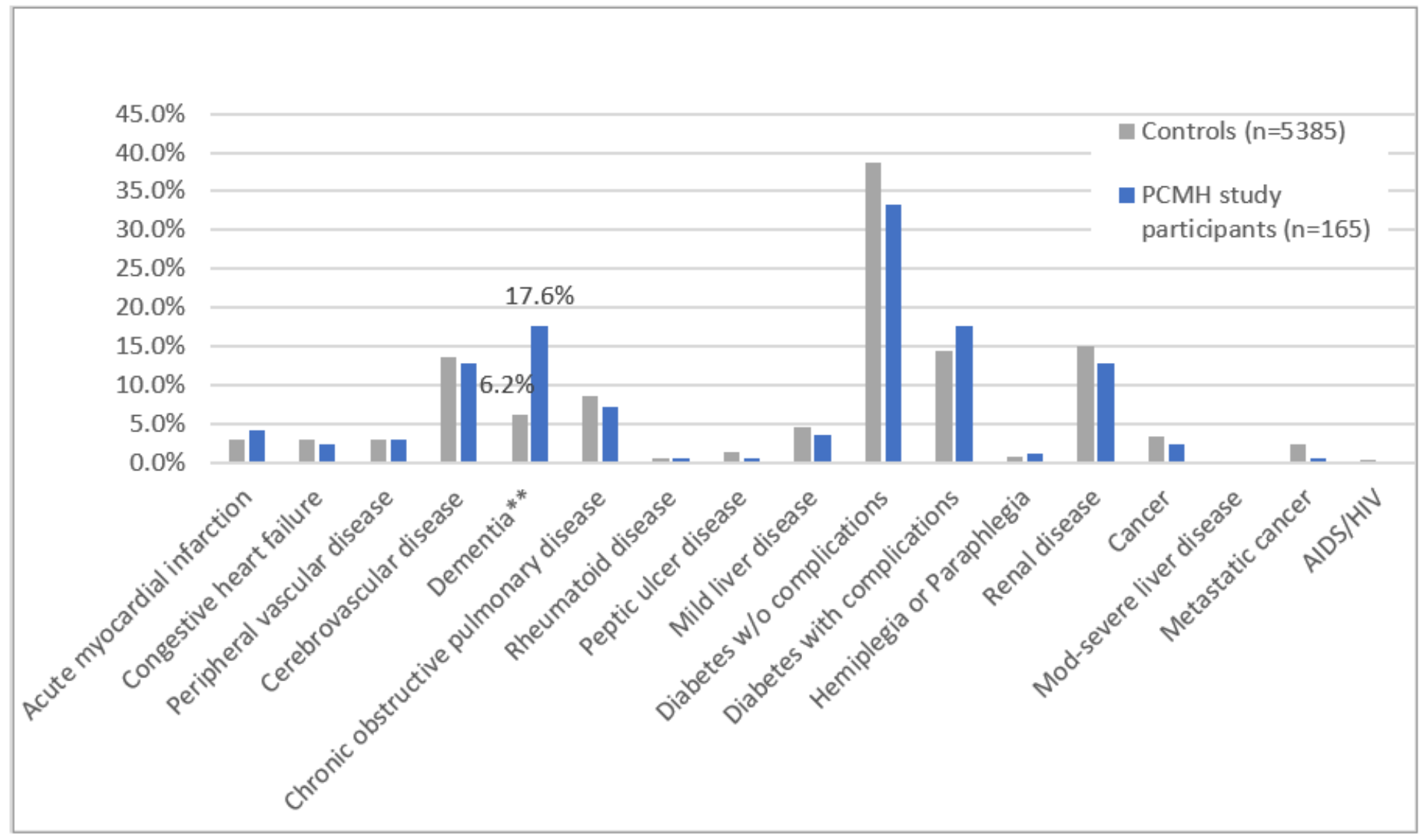

Figure 2

Post-matching case-control prevalence of comorbidities

Weighted chi-square tests conducted to assess if differences were statistically-significant. p-value: ** $<0.01, *<0.05, \wedge<0.1$ 


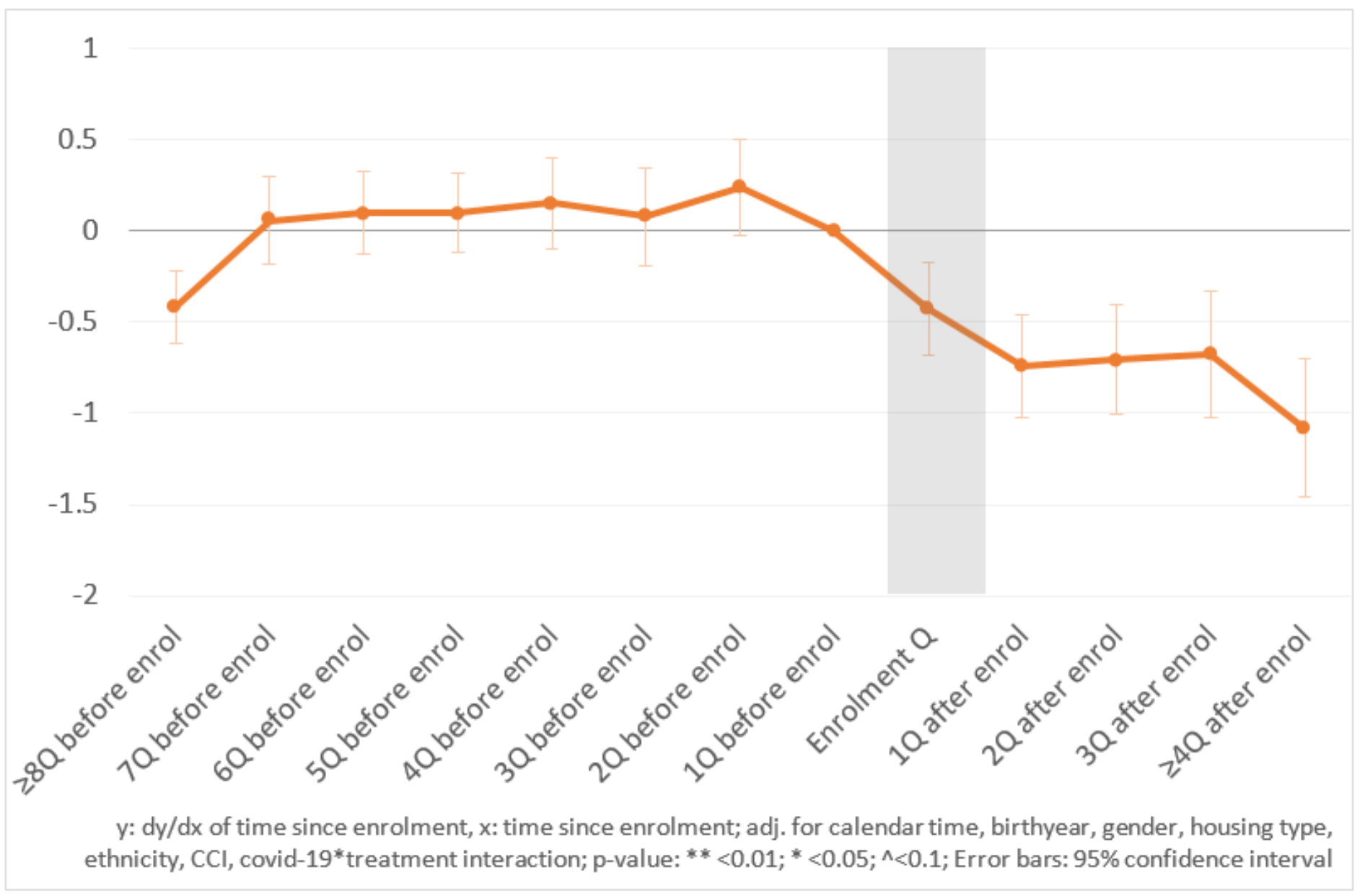

\section{Figure 3}

Polyclinic visits two-part model (Combined), difference-in-difference:

Difference in no. of polyclinic visits per person-quarter between PCMH study participants and controls, by time since enrolment 


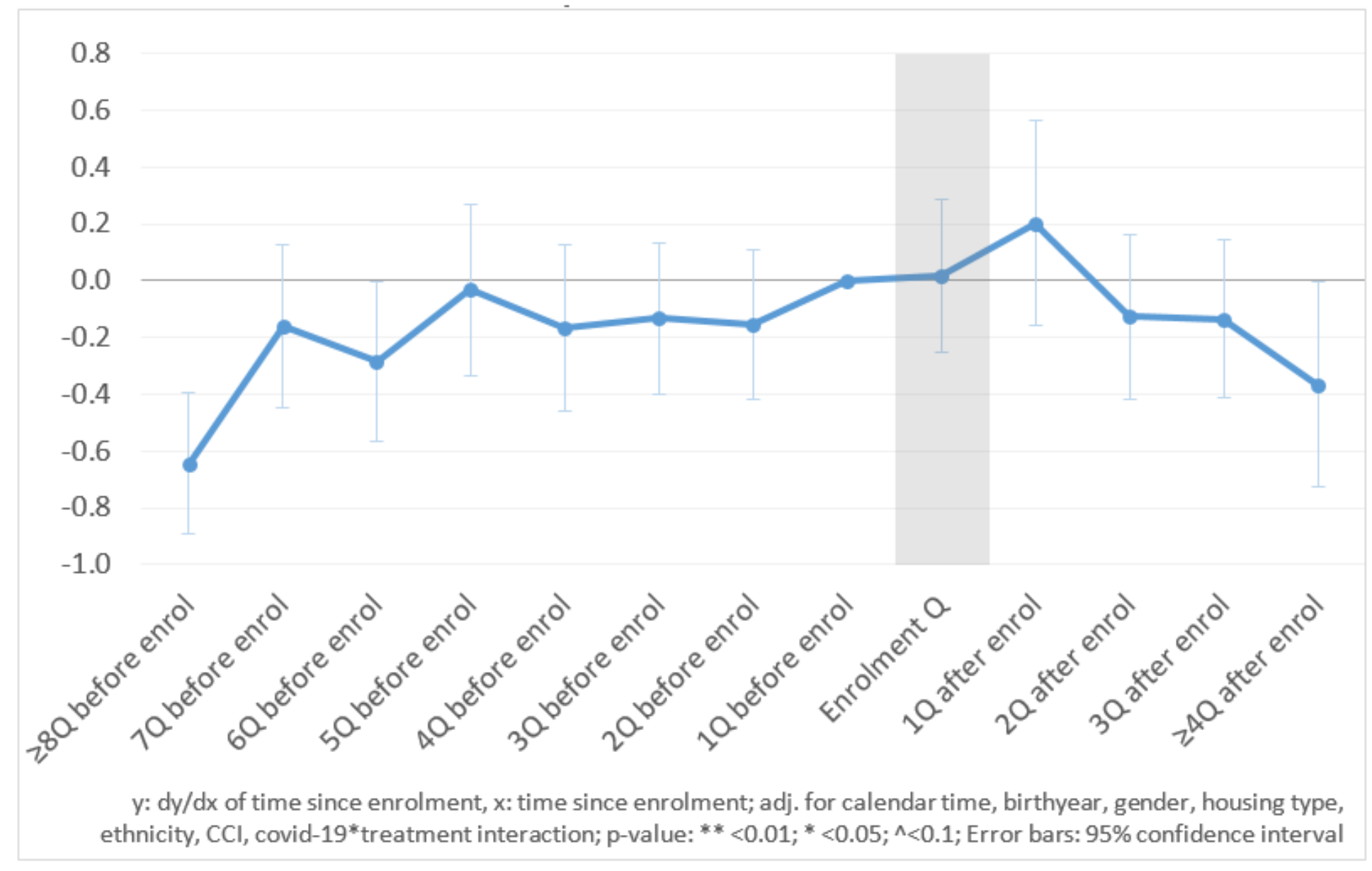

\section{Figure 4}

visits two-part model (Combined), difference-in-difference:

Difference in no. of SOC visits per patient-quarter between PCMH study participants and control patients, by time since enrolment 


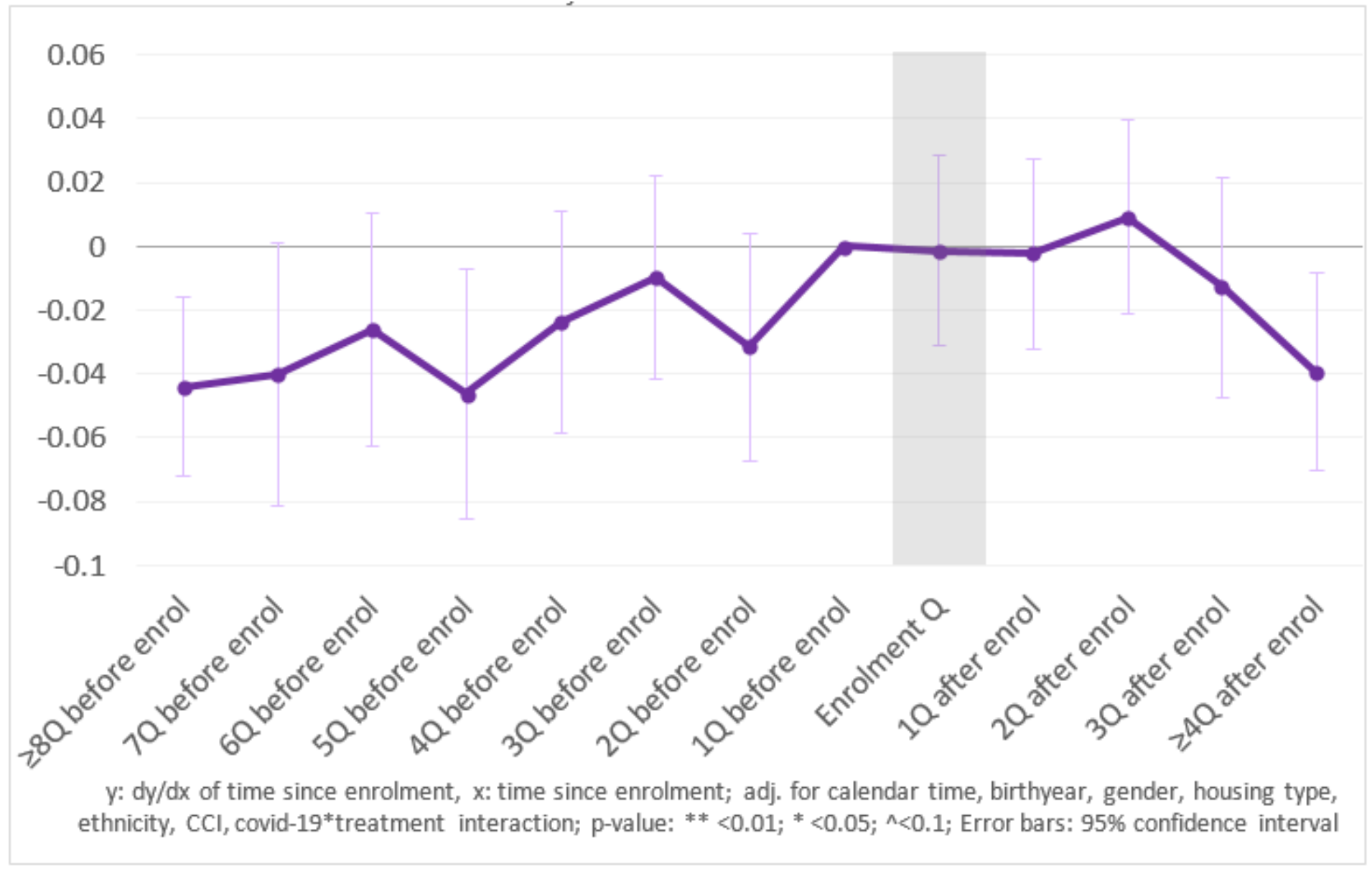

\section{Figure 5}

Emergency department visits two-part model (Combined), difference-in-difference:

Difference in no. of ED visits per patient-quarter between PCMH study participants and control patients, by time since enrolment 


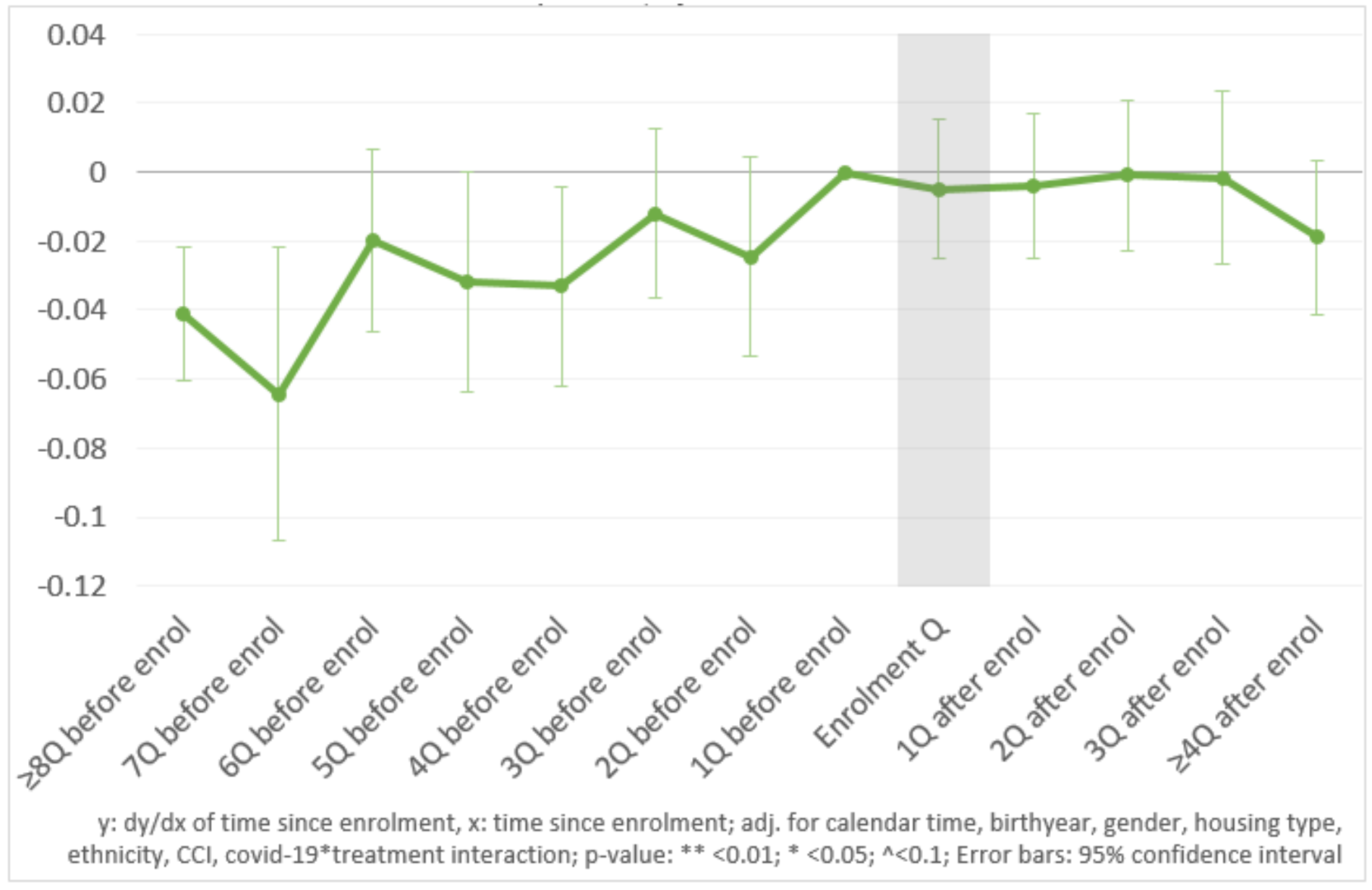

\section{Figure 6}

Inpatient admissions two-part model (Combined), difference-in-difference:

Difference in no. of inpatient admissions per person-quarter between PCMH study participants and control patients, by time since enrollment

\section{Supplementary Files}

This is a list of supplementary files associated with this preprint. Click to download.

- AppendixTableTwo.docx 